\title{
Assessment of retinoblastoma RNA reflux after intravitreal injection of melphalan
}

\author{
Ursula Winter, ${ }^{1,2}$ Michael Nicolas, ${ }^{3}$ Mariana Sgroi, ${ }^{4}$ Claudia Sampor, ${ }_{1}^{5}$ Ana Torbidoni, ${ }_{1}^{2}$ \\ Adriana Fandiño, ${ }^{4}$ Guillermo L Chantada, ${ }^{2,5}$ Francis L Munier, ${ }^{3}$ Paula Schaiquevich ${ }^{1,2}$
}

\begin{abstract}
${ }^{1}$ Unit of Clinical
Pharmacokinetics, Hospital de Pediatría JP Garrahan, Buenos Aires, Argentina

${ }^{2}$ National Scientific and Technical Research Council, CONICET, Buenos Aires, Argentina

${ }^{3}$ Department of Ophthalmology, University of Lausanne, JulesGonin Eye Hospital, Lausanne, Switzerland

${ }^{4}$ Department of Ophthalmology, Hospital de Pediatría JP Garrahan, Buenos Aires, Argentina

${ }^{5}$ Department of HematologyOncology, Hospital de Pediatría JP Garrahan, Buenos Aires, Argentina
\end{abstract}

\section{Correspondence to}

Dr Paula Schaiquevich, Unit of Clinical Pharmacokinetics, Hospital de Pediatría J.P. Garrahan, Combate de los Pozos 1881, C1245AAL, Buenos Aires, Argentina; paula.schaiquevich@ gmail.com

Received 6 April 2017 Revised 12 September 2017 Accepted 18 October 2017

Published Online First

31 October 2017

\section{ABSTRACT \\ Background Intravitreal injection of chemotherapy in retinoblastoma eyes with vitreous seeds may lead to a risk of extraocular tumour dissemination that has not been assessed so far.}

Aims To develop a sensitive and clinically feasible technique to assess for potential retinoblastoma cell reflux after intravitreal injection of melphalan.

Methods Filter papers were cut in $6 \mathrm{~mm}$ diameter circles and sterilised before use. Eyes with retinoblastoma vitreous seeds (group D, International Classification) received weekly intravitreal melphalan injections $(20 \mu \mathrm{g}$ or $30 \mu \mathrm{g} /$ dose) followed by cryotherapy as part of local treatment. Immediately after finishing the injection and cryotherapy, filter papers were placed on the injection site and on the cryoprobe tip to assess for the expression of the cone-rod homeobox gene (CRX) by real-time qPCR as a surrogate of retinoblastoma RNA. The assay was developed and validated to determine sensitivity, linearity, recovery, repeatability and reproducibility.

Results The assay for quantitation of CRX expression was linear in the range of 1 to 1000 cells. The lowest limit of detection was one retinoblastoma cell and allowed to recover $100 \%$ of the cell load in external supplementation. A total of 14 eyes received 22 cycles of intravitreal melphalan and were evaluated for potential extraocular tumour cell dissemination using the developed technique. None of the cycles were positive for CRX in samples from the scar or from the cryoprobe tip.

Conclusions A sensitive and simple method of tumour cell assessment has been developed that can be used in the clinics to assess for potential extraocular dissemination after intravitreal injections to assure its performance.

\section{INTRODUCTION}

Management of eyes with retinoblastoma vitreous seeds has changed over the last 5 years allowing to save eyes that previously were enucleated upfront. ${ }^{1}$ Currently, a fundamental part of conservative treatment for intraocular retinoblastoma with vitreous seeds is the use of intravitreal injections of chemotherapeutic agents followed by cryotherapy. ${ }^{2}$ The most important advantages of intravitreal chemotherapy include intraocular tumour control and a low incidence of local and systemic adverse events. Despite initial attempts to perform intravitreal injections of chemotherapy or gene therapy to target vitreous seeds, this route of drug delivery has not been incorporated in the routine of retinoblastoma treatment ${ }^{3-5}$ One of the main concerns that has delayed a broad use of intravitreal injections in eyes with retinoblastoma is the possibility of extraocular tumour dissemination from the needle tract. ${ }^{3}$

Based on the potential complication of orbital seeding, Munier et al proposed a modified technique of intravitreal chemotherapy. ${ }^{67}$ For a safe intervention, several recommendations have been reported for ophthalmologists, including evaluation of tumour-free injection sites using ultrasound biomicroscopy whenever the visualisation of the entry site is compromised due to opaque media or insufficient dilation, anterior chamber paracentesis and the application of three consecutive cycles of cryotherapy. ${ }^{7}$ Nonetheless, although the procedure has been used in a relatively high number of patients, there is no technique that allows to assess for potential tumour dissemination at a microscopic level. ${ }^{9}$ Therefore, the aim of our study was to develop a sensitive and practical technique to assess for the reflux of tumour RNA, as a surrogate marker for potential tumour seeding on the needle tract after the intravitreal injection of chemotherapeutic agents in patients with retinoblastoma treated at reference centres in Argentina and Switzerland.

\section{METHODS}

\section{Development and validation of the RT-qPCR} assay

In a laminar flow hood, $6 \mathrm{~mm}$ diameter circular filter paper pieces (Whatman42, Sigma-Aldrich) were obtained using sterile scissors (figure 1A). Each paper was exposed to UV light for $30 \mathrm{~min}$ on each side and placed in autoclave bags for sterilisation (one cycle of $120^{\circ} \mathrm{C}$ at $1 \mathrm{~atm}$ of pressure).

To construct the calibration curves, a commercial retinoblastoma cell line, WERI-RB1 (ATCC HTB-169, Manassas, Virginia), was used for RNA isolation. Briefly, after assessing for cell-culture viability using trypan blue, RNA was extracted from $10^{6}$ WERI-Rb1 cells using PureLink RNA Mini Kit (Invitrogen) and resuspended in $30 \mu \mathrm{L}$ of RNAse-free water. Then, $6 \mu \mathrm{L}$ was separated to obtain cDNA by retrotranscription (Superscript III First-Strand Synthesis SuperMix) achieving a final volume of $20 \mu \mathrm{L}(10000$ cells $/ \mu \mathrm{L})$. Serial dilutions were prepared using Milli-Q water. The transcription levels of the lineage-specific gene marker cone-rod homeobox (CRX) and GAPDH (housekeeping gene) were determined by quantitative real-time PCR (RT-qPCR) using SYBR Green Power Master Mix (Life technologies) and a 7500 real-time PCR system (Applied Biosystems). ${ }^{10}{ }^{11} \mathrm{CRX}$ was considered positive in 
A.

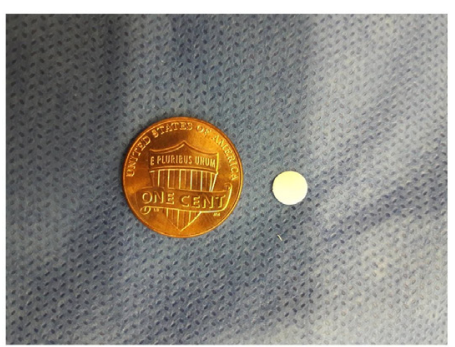

B.

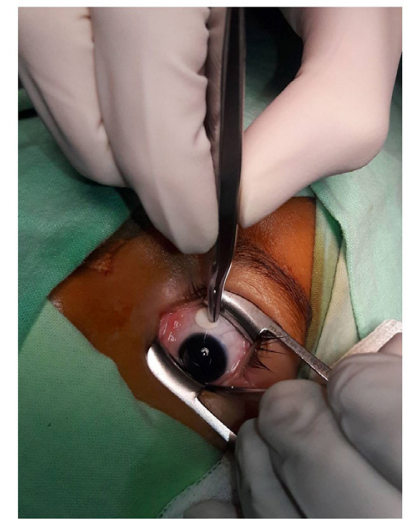

Figure 1 (A) An image of the filter paper used for each intervention compared with the size of a dime, (B) during the intravitreal injection followed by cryotherapy and immediately after placement of the filter paper on the scar.

a sample if each replica of the threshold cycle $(\mathrm{Ct})$ and that of the housekeeping gene was $<40$ and the SD between replicas was $<0.5$.

Freeze-thaw cycles were evaluated to enable extraction of RNA from retinoblastoma cells recovered from filter paper. In this case, filter papers supplemented with different quantities of WERI-Rb cells (1 to 1000 cells/paper) were placed in $0.2 \mathrm{~mL}$ thin-wall tubes in sextuplicate and treated as follows: two freeze-thaw cycles consisting of freezing at $-80^{\circ} \mathrm{C}$ for 24 hours and afterwards $15 \mathrm{~min}$ in an ice bath were performed. Then, RT-qPCR was performed as previously described and the amount of cDNA obtained from the cells was interpolated in the calibration curve. ${ }^{10} 11$

In order to assay if the filter paper adsorbed retinoblastoma cell cDNA interfered with the quantitation, $6 \mu \mathrm{L}$ of three cDNA dilutions was added to filter papers in triplicate, processed and the amount of cDNA was interpolated in the calibration curve obtained from filter paper-free samples.

PCR amplification efficiency and linearity was determined using freshly prepared dilutions of cDNA covering the range between 1 and 1000 retinoblastoma cells. Each dilution was analysed in duplicate, and three runs were performed on different days to assay for reproducibility and inter-run precision (defined as the percent coefficient of variation of Cqs of each sample level between independent runs). A calibration curve was accepted if the slope was between -3.9 and -2.9 corresponding to $80 \%$ to $120 \%$ PCR efficiencies and if the correlation coefficient $\left(\mathrm{r}^{2}\right)$ of the curve was $\geq 0.97$. The efficiency of the RT-qPCR reaction was calculated from the slope of the calibration curve using the formula $\% \mathrm{E}=\left(10^{(-1 / \text { slope })}-1\right) \times 100$.

The sensitivity of the method was defined as the limit of quantitation based on the lowest number of cells that could be detected in six replicates assayed in two different runs with adequate precision.

Reproducibility of the assay was determined by performing the described assay at three levels of cell concentration in triplicate on three different days. The threshold cycle $(\mathrm{Ct})$ was recorded for each sample.

Finally, the method transferability was evaluated by preparing and sending to different centres four blind samples-one negative and three positive-for analysis.

\section{Clinical sample processing and application}

Eyes with retinoblastoma seeds that were considered for intravitreal chemotherapy were first assessed using ultrasound biomicroscopy (Ophthalmic Technologies, Canada), to identify a tumour-free meridian suitable for the injection. Transient hypotony was obtained by anterior chamber paracentesis aspirating 0.1 or $0.15 \mathrm{~mL}$ depending on the volume of melphalan solution to be intravitreally administered. Between 2.5 and $3.5 \mathrm{~mm}$ from the limbus, a $33 \mathrm{G}$ needle mounted on a tuberculin syringe was introduced to the centre of the pupillary area immediately behind the lens under microscope viewing. ${ }^{7}$ Then, 20 or $30 \mu \mathrm{g}$ of melphalan dissolved in 0.1 to $0.15 \mathrm{~mL}$ of saline was injected followed by cryotherapy (three freeze-thaw cycles) at the entry site during needle removal. Afterwards, a sterile filter paper held with a sterile forceps was placed on the scar left by the injection for $10 \mathrm{~s}$ (figure 1B). Simultaneously, another paper was placed on the cryoprobe tip and both papers were placed in separate tubes for immediate processing as previously described. A total of $6 \mu \mathrm{L}$ of PBS was added to each tube.

Finally, ocular status of the affected eye was assessed with fundus photography and clinical control at presentation and after starting the local treatment. Additionally, the eyes were evaluated using RetCam (Clarity, Pleasanton, California, USA).

The study protocol and informed consent were approved by the Institutional Review Boards of both Hospital de Pediatria JP Garrahan, Argentina (Protocol \# 857) and Jules-Gonin Eye Hospital, Switzerland (authorisation \#2016-00149).

\section{RESULTS}

Primer specificities were confirmed by melting curve analysis that showed single peaks.

First, we evaluated if freeze-thaw cycles were an adequate method to recover RNA from retinoblastoma cells adsorbed in filter paper. The results showed a median (range) recovery of 98.5\% (94.1-103.4) compared with RNA recovered from cell suspension using freeze-thaw cycles as well.

In order to avoid inaccurate cell counting and having established that almost $100 \%$ of cell RNA could be extracted with freeze-thaw cycles, we evaluated the potential adsorption of cDNA to filter paper compared with external solution in water. The median (range) recovery was 100.3\% (96.5-107.0) supporting the use of filter paper.

A representative amplification plot of a calibration curve for CRX is shown in figure 2A. Based on these plots and the precision calculated for each level of the calibration curve, the detection limit for the assay expressed as sensitivity was estimated at one retinoblastoma cell at an average $\mathrm{Cq}$ value $<40$ cycles. The precision or \% $\mathrm{CV}(\mathrm{SD})$ for the limit of detection set at one cell was $1.26 \%(0.44)$.

A strong linear relationship could be established by plotting Cq values against the $\log 10$ number of retinoblastoma cells in the range of 1 to 1000 cells (figure 2B).

Low intrarun variability was observed for all four analysed levels with a median (range) value of 1.5\% (0.34-2.46). Assay reproducibility was also found to be high as a low median (range) $\% \mathrm{CV}$ inter-run value of $2.66 \%(1.16-3.76)$ was obtained. Therefore, for all assayed cDNA levels $\% \mathrm{CV}$ was $<5 \%$ indicating high reproducibility.

A total of four samples were sent to clinical centres to test for transferability. Centres were blind to the concentration of cDNA provided and processed as described. Consistent results were obtained as 4/4 samples were quantified accurately. 
A.

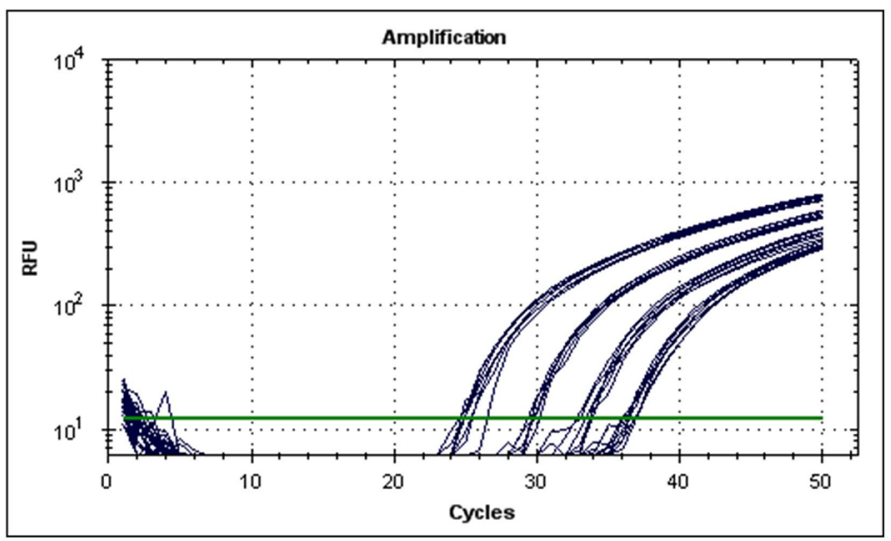

B.

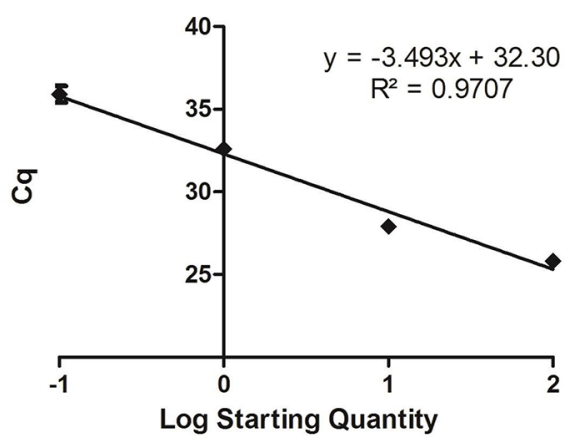

Figure 2 (A) Representative amplification plot showing from left to right the curves of decreasing concentrations of cDNA extracted from commercial retinoblastoma cells. (B) Standard curve for CRX assessment (generated from the amplification plots) built with the Cq values against the $\log$ number of $c D N A$. The best fit is shown as a solid line and the $r^{2}=0.98$.

The applicability of the technique was evaluated in a set of clinical samples obtained after intravitreal injection of chemotherapy into eyes with intraocular retinoblastoma and vitreous seeds. A total of 22 cycles of intravitreal melphalan administered to 14 eyes of patients with unilateral retinoblastoma were evaluated. In all cases, samples collected from the injection wound or the cryoprobe tip were CRX negative.

\section{DISCUSSION}

Conservative treatments of eyes with retinoblastoma have greatly evolved over the past 5 years. Local treatment of retinoblastoma using serial intravitreal administrations of chemotherapy may disrupt ocular barriers potentially leading to extraocular seeding of tumour cells. Thus, assessment of cellular reflux may be helpful to determine whether a safe procedure has been performed. Tumour spread cannot be assessed using current imaging techniques and a method of cell assessment in potential vitreous reflux has never been developed. The only previous report consisted of cytopathological evaluation of the water recovered after submersion of the eye. ${ }^{9}$ Therefore, we developed a sensitive, precise, linear, reproducible and clinically feasible technique of tumour cell assessment to quantitate for potential reflux.

Recent studies have shown that CRX, a lineage biomarker of photoreceptors, is expressed in the outer nuclear layer of the normal human retina corresponding to cone-and-rod photoreceptor cell distribution in the retina and retinoblastoma. ${ }^{11-13}$ Therefore, we used this biomarker to develop a sensitive technique that allows detection of as few as one retinoblastoma cell with very good precision. Since CRX is not a tumour marker, it is not specific for malignancy and, potentially, normal photoreceptor cells that may reflux could yield a false-positive result. ${ }^{12} 13$ However, there were no positive cases in our study showing the effectiveness of this technique for intravitreous injection in terms of avoiding cell reflux. Additionally, false-positive results from normal photoreceptor cells would be highly unlikely considering the entry site. In this study, a positive control of the technique was included by adding retinoblastoma cells or RNA extracted from these cells to filter papers for quantitation by RT-qPCR. As shown, the method resulted linear in the evaluated range with marked sensitivity. Moreover, although it would be extremely useful to assess the developed technique in an in vivo animal model, the currently available tumour-bearing animal models are xenograft mice that do not allow for replicating the intravitreal technique as performed in humans due to anatomical restrictions. ${ }^{10}$ Noteworthy, cell recovery from filter paper was almost $100 \%$ at different cell loads with no interference from paper components.

This simple technique using filter paper in order to adsorb the cells may also be helpful in the clinics to evaluate reflux in difficult cases. ${ }^{14}$

Microscopical dissemination is theoretically possible in each of the different steps of the procedure of intravitreal injection. In an experimental model of intravitreal injection without prior paracentesis, $<5 \%$ of the original $50 \mu \mathrm{L}$ injection was found lost to reflux. ${ }^{15}$ According to the technique proposed by Munier et al, at the moment of withdrawing the needle, a triple freeze cryotherapy is applied to seal the needle tract and to destroy malignant cells following a contaminated needle or egressing by reflux. ${ }^{67}$ Our study aimed to evaluate reflux after completing the ophthalmological procedure of intravitreal injection currently performed. We did not attempt to evaluate if reflux could occur before the cryotherapy is applied in order not to modify the sequence and timings of the procedure. In the present cohort of eyes, no retinoblastoma cells were detected on the needle tract left by the injection or on the cryoprobe tip used for cryotherapy following the injection. Moreover, no patient showed orbital relapse supporting the absence of tumour cell spread after intravitreal injection. This study design could be regarded as a limitation and thus, we support the performance of future studies to assess the composition of vitreous reflux including retinoblastoma cell RNA. Nonetheless, a specific and feasible study design should be performed without compromising patient safety.

A possible limitation for the transferability of the method is the availability of the RT-qPCR technique at clinical centres. In most clinical centres where intravitreal injections for retinoblastoma treatment are performed, RT-qPCR is currently performed for molecular diagnosis on a daily basis. Nonetheless, training in general laboratory skills and in molecular biology is required in order to perform a correct technique. Alternatively, samples could be obtained and partially processed at most clinical centres and thereafter sent to more specialised centres to perform RT-qPCR on a collaborative basis. 
We conclude that, at least in the context of a pre-injection anterior chamber paracentesis, no reflux of tumour cells could be detected by this sensitive and relatively easy-to-implement technique, after intravitreal injection of melphalan for the treatment of retinoblastoma with vitreous seeding.

Contributors UW and PS conceived and designed the study. UW and MN developed and validated the molecular biology technique. UW, AT and MN processed the samples. MS, AF, CS and FM participated in subject enrolment and sample collection. GLC, PS and FM designed the study and wrote the paper. All authors contributed to the review of the literature, in drafting the manuscript and in approving the final manuscript.

Funding This work was funded by the National Institute of Cancer, Ministry of Health of Argentina, Fund for Ophthalmic Knowledge, New York, USA; Fundación Natalie D Flexer de Ayuda al Niño con Cáncer.

Competing interests None declared.

Patient consent Guardian consent obtained.

Ethics approval Institutional Review Boards of both Hospital de Pediatria JP Garrahan, Argentina (Protocol \# 857) and Jules-Gonin Eye Hospital, Switzerland (authorisation \#2016-00149)

Provenance and peer review Not commissioned; externally peer reviewed.

(c) Article author(s) (or their employer(s) unless otherwise stated in the text of the article) 2018. All rights reserved. No commercial use is permitted unless otherwise expressly granted.

\section{REFERENCES}

1 Munier FL. Classification and management of seeds in retinoblastoma. Ellsworth lecture ghent august 24th 2013. Ophthalmic Genet 2014;35:193-207.

2 Francis JH, Abramson DH, Gaillard MC, et al. The classification of vitreous seeds in retinoblastoma and response to intravitreal melphalan. Ophthalmology 2015:122:1173-9.
3 Suzuki S, Aihara Y, Fujiwara M, et al. Intravitreal injection of melphalan for intraocular retinoblastoma. Jpn J Ophthalmol 2015;59:164-72.

4 Chévez-Barrios P, Chintagumpala M, Mieler W, et al. Response of retinoblastoma with vitreous tumor seeding to adenovirus-mediated delivery of thymidine kinase followed by ganciclovir. J Clin Oncol 2005;23:7927-35.

5 Kivelä T, Eskelin S, Paloheimo M. Intravitreal methotrexate for retinoblastoma. Ophthalmology 2011;118:1689-1689.e6.

6 Munier FL, Gaillard MC, Balmer A, et al. Intravitreal chemotherapy for vitreous disease in retinoblastoma revisited: from prohibition to conditional indications. $\mathrm{Br} J$ Ophthalmol 2012;96:1078-83.

7 Munier FL, Soliman S, Moulin AP, et al. Profiling safety of intravitreal injections for retinoblastoma using an anti-reflux procedure and sterilisation of the needle track. $\mathrm{Br}$ J Ophthalmol 2012;96:1084-7.

8 Shields CL, Douglass AM, Beggache M, et al. Intravitreous chemotherapy for active vitreous seeding from retinoblastoma: outcomes after 192 consecutive injections. The 2015 howard naquinAssociation of Cone-Rod Homeobox Transcription Factor Messenger RNA With Pediatric Metastatic Retinoblastoma lecture. Retina 2016:36:1184-90.

9 Francis JH, Xu XL, Gobin YP, et al. Death by water: precautionary water submersion for intravitreal injection of retinoblastoma eyes. Open Ophthalmol J 2014;8:7-11.

10 Pascual-Pasto G, Olaciregui NG, Vila-Ubach M, et al. Preclinical platform of retinoblastoma xenografts recapitulating human disease and molecular markers of dissemination. Cancer Lett 2016;380:10-19.

11 Torbidoni AV, Laurent VE, Sampor C, et al. Association of cone-rod homeobox transcription factor messenger ma with pediatric metastatic retinoblastoma. JAMA Ophthalmol 2015;133:805-12.

12 Santagata S, Maire CL, Idbaih A, et al. CRX is a diagnostic marker of retinal and pinea lineage tumors. PLoS One 2009:4:e7932.

13 Glubrecht DD, Kim J-H, Russell L, et al. Differential CRX and OTX2 expression in human retina and retinoblastoma. J Neurochem 2009:111:250-63.

14 Seregard S, Singh AD. Retinoblastoma: direct chemotherapeutic drug delivery into the vitreous cavity. Br J Ophthalmol 2012:96:473-4.

15 Brodie FL, Ruggiero J, Ghodasra DH, et al. Volume and composition of reflux after intravitreal injection. Retina 2014:34:1473-6. 